\title{
Customer Interaction Management with SCM
}

\author{
Jörg Schuster \\ Center for Information \\ and Language Processing (CIS) \\ Ludwig Maximilian University of Munich \\ (LMU), Germany
}

\author{
Matthias Bargel \\ Center for Information \\ and Language Processing (CIS) \\ Ludwig Maximilian University of Munich \\ (LMU), Germany
}

\begin{abstract}
SCM is a simple, modular and flexible system for web monitoring and customer interaction management. SCM is completely web based. It combines all technologies, data, software agents and human agents involved in the monitoring and customer interaction process. It can be used for messages written in any natural language. Although the prototype of SCM is designed for classifying and processing messages about mobile phone related problems in social networks, SCM can easily be adapted to other text types such as discussion board posts, blogs or emails. Unlike comparable systems, SCM uses linguistic technologies to classify messages and recognize paraphrases of product names. For two reasons product name paraphrasing plays a major role in SCM: First, product names typically have many, sometimes hundreds or thousands of intralingual paraphrases. Secondly, product names have interlingual paraphrases. The same products are often called or spelt differently in different countries and/or languages. By mapping product name variants to an international canonical form, SCM allows for answering questions like "Which statements are made about this mobile phone in which languages/in which social networks/in which countries/. . . ?”
\end{abstract}

\section{Introduction}

More than 1.145 million people worldwide use social networking sites such as Facebook, Twitter, MySpace or Foursquare (see http://www.radicalgeography.co.uk/brandnewworldcar dsort.doc). Therefore social media have to be explored as an additional communication channel between customers and companies. In June 2011, there were more than 19 million active Facebook users in Germany (see http://www.socialbakers.com/facebook- statistics/). Therefore, some German companies especially in the telecommunication sector - already have regularly updated Facebook pages for customer service and support, e.g. Telekom (German Telecom, see http://www.facebook.com/telekomhilft), Vodafone (see http://www.facebook.com/vodafoneDE), O2 (see http://www.facebook.com/o2) or Nokia (see http://www.facebook.com/nokiahomebase).

For the traditional communication channels such as telephone, fax, letter, email and online live chat, there are already established ways of dealing with incoming requests. They are usually handled in contact centers. Contact center agents assign customer requests to internal business processes. However, social networking has not yet been integrated into customer interaction management tools. In order to complement their current marketing and business development activity with social media and other online marketing activities, companies expand their focus on social networks as well as on textual data such as RSS feeds, emails, blogs, etc. Until now, the dialog with customers is still one-sided and not designed as a twoway process addressing the customer on the same communication channel.

Our proposed solution towards a web monitoring and customer interaction management system is quite simple. We focus on a modular architecture fully configurable for all components integrated in its workflow (e.g. software, data streams, human agents for customer service). Although the prototype of SCM is designed for processing customer messages posted on social networking sites about mobile phone specific issues, it can easily be adapted to other topics and to other text types such as emails, blogs, etc. Unlike the commercial monitoring systems mentioned below, we use a linguistic, rule-based approach for message classification and product name recognition. Our approach can easily be combined with statistical methods, though. One of the most salient features of 
SCM is its paraphrasing module for intra- and interlingual variants of product names and similar entities (such as tariffs, manufacturers and providers). SCM has two types of independent agents to classify customer messages: content and sentiment classifiers. They basically consist of a set of positive and a set of negative constraints. If one of the positive constraints holds, the classifier's tag is assigned. If one of the negative constraints holds, the classifier's tag is not assigned, irrespective of the positive constraints. Moreover, SCM can be used with any natural language. In our prototype instance of SCM, we created classifiers and product names for three very different languages: German, Greek and Korean.

The paper is structured as follows. In the next section we discuss some related work. In Section III we give an overview of the system architecture. In Section IV we explain how our named entity paraphrasing engine works. Section $\mathrm{V}$ is about how the grammars of SCM's classification agents are developed and applied.

\section{Related Work}

As SCM (Socialcom Customer Interaction Management) is a system that was created in a collaborative project (called Socialcom) between CIS (Center for Information and Language Processing, an Institute of the University of Munich (LMU)) and the Munich based company Telenet $\mathrm{GmbH}$ Kommunikationssysteme, i.e. as it was created in a collaborative project between research and industry, two types of related work must be considered. Previous work can, on one hand, be found in scientific articles about text classification and named entity normalization. On the other hand, the systems of the various companies that offer customer interaction management and web monitoring must also be considered to be previous work.

There are a plenty of commercial solutions, of varying quality, for these tasks including GoogleAlerts by Google (see http://www.google.com/alerts), BuzzStream by BuzzStream (see http://www.buzzstream.com/), Sysomos by Sysomos Inc. (see http://www.sysomos.com/), Alterian by Alterian (see http://www.alterian.com/), Visible Technologies by Visible Technologies (see http://www.visibletechnologies.com/) and Radian6 by Radian6 Technologies Inc. (see http://www.radian6.com/). The current trend is to develop virtual contact centers that process the costumers' messages and forward them to the company's service and support team. Eptica by EADS e.g. provides a commercial tool for customer interaction management via Facebook (see http://www.eptica.com/). Other systems try to predict election results (see [1]) or success of movies and music (see [2]) by using scientific analysis of opinion polls or by doing sentiment analysis on special web blogs or online forum discussions. Further relevant issues are topic and theme identification and sentiment detection. Since blogs consist of news or messages dealing with various topics, blog content has to be divided into several topic clusters. Pal and Saha (see [3]) from Hewlett Packard developed the "Best Separators Algorithm" which cuts documents into blocks according to theme relevance.

As scientific articles are concerned, there are basically two topics that are relevant to our work: Text classification and named entity normalization. There does not seem to be much literature about the classification of texts of the type we dealt with. An interesting description of a blog classification system (blogs are much closer in many respects to the texts we classified than most other text types) is given in [4]. Gilad Mishne developed "a method for tagging blog posts based on a collaborative filtering approach: given a blog post, tags for it are suggested according to tags assigned to other, similar posts” ([4], page 101). As the tags that our texts are supposed to receive usually strongly depend on "named entities" (such as product or tariff names) and other strings that occur in them, we used a different approach.

As concerns proper name or named entity normalization, two articles are especially interesting: Jijkoun et alii discuss a method for "named entity normalization in user generated content" in [5]. After giving an overview of the literature about named entity normalization ([5], page 24), they discuss "five improvements to [a] baseline NEN [i.e. named entity normalization] algorithm" ([5], page 23). One of their ideas is to use anchor texts in Wikipedia articles to find variants of named entities. In [6], Hema Raghavan and James Allan compare seven (mostly statistical) methods for normalizing proper name variants. For two reasons, we chose to use an approach that differs from Raghavan's and Allan's techniques as well as from the approach used by Jijkoun et alii: First the variants of product and tariff names usually follow rather obvious and regular patterns. We felt it would be unnecessarily complicated to use statistical methods for them. Secondly, the variants of the names of mobile phone related products and tariffs can not normally be found in Wikipedia. 


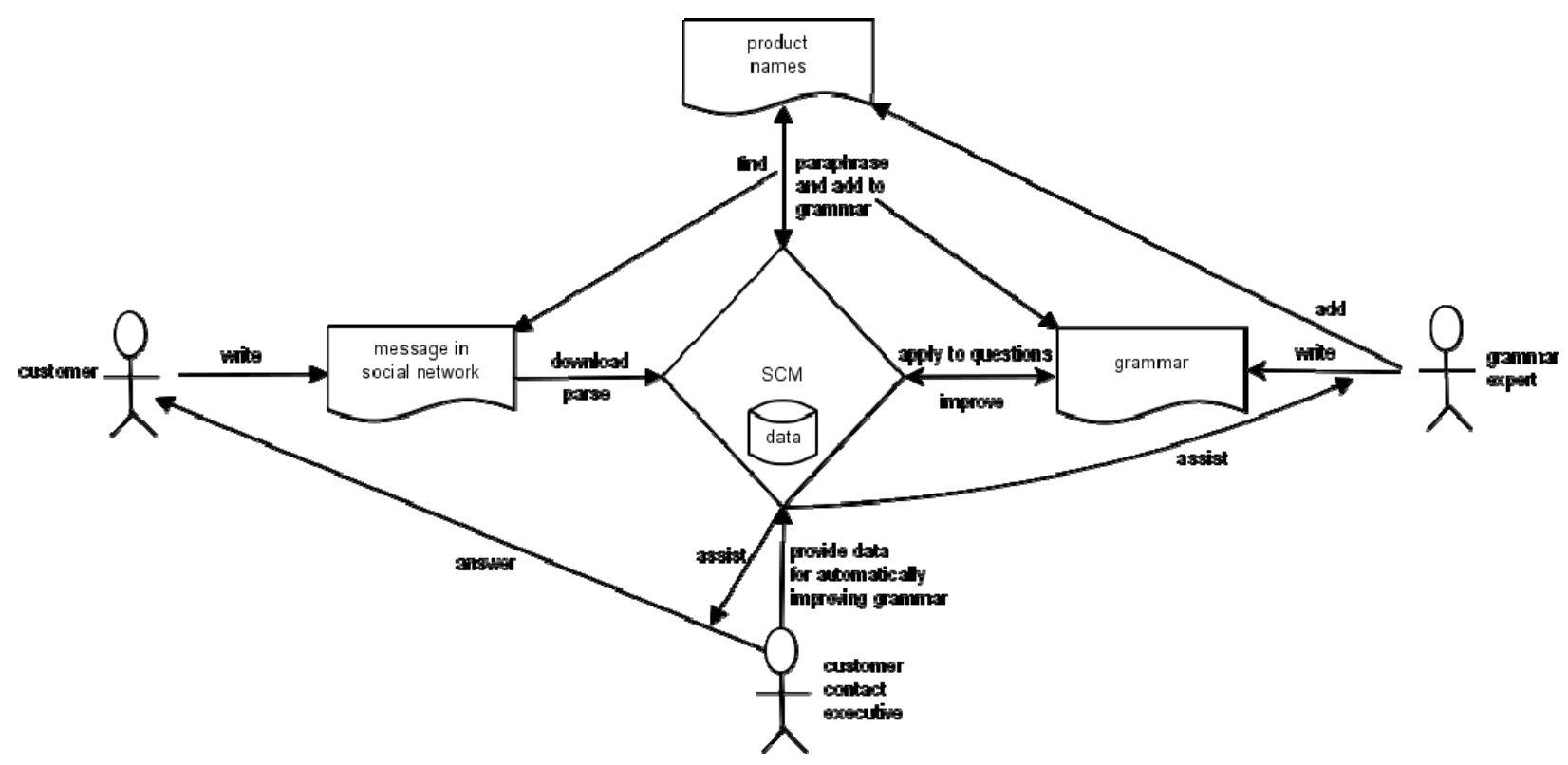

Figure 1. The basic design of SCM

\section{System Overview}

SCM is a web based monitoring and customer interaction management system that combines all technologies, data, software agents and human agents involved in the monitoring and customer interaction process, such as the technologies used for downloading, processing and storing data, the software agents used for classification and similar tasks and the human agents such as grammar experts and contact center agents. Figure 1 gives an overview of SCM's design: Customers post messages in social networks and other web sites. Messages typically concern products and/or services sold or provided by a company. Most messages state that a problem of a certain type occurred with a certain product or service. Some messages also contain expressions of praise or thank. SCM downloads all messages and stores them in a normalized format. It applies its grammar to the messages in order to recognize all product names in it and in order to assign a set of content tags (such as hotline or delivery or malfunction) and a sentiment tag (at the moment either positive or negative). (We will say immediately what we understand by a grammar.) The message, their tags and the list of product names are then forwarded to those contact center agents that are experts for the recognized type of problem. If a message has been assigned a wrong tag or no tag at all, contact center agents can assign a tag manually. They have to mark those parts of the message that justify its being assigned the tag. Thus, contact center agents unknowingly add new constraints to the grammar.
Actually, SCM does not only contain one grammar. It contains as many grammars as there are classifier objects. Each classifier has a (content or sentiment) tag, a set of positive and a set of negative constraints. (Figure 3 shows a simple content classifier.) If one of the positive constraints holds, the classifier's tag is assigned. If one of the negative constraints holds, the classifier's tag is not assigned, irrespective of the positive constraints. I.e. negative constraints can be used to override positive constraints.

Put simply, the constraints are regular expressions. Yet, as they can contain special terms that refer to other grammars (such as \mobile_phone), it would be more adequate to call them Local Grammars in the sense of [7]. Each instance of SCM comes with a predefined set of such special terms. (The idea is to tailor new instances of SCM to the needs of the companies or organizations that use it.) Grammar experts cannot add special terms or change their meaning. They just use them: If, e.g., a grammar line contains foo \mobile_phone bar, it will match foo, followed by a space character, followed by any variant of any mobile phone name known to the used instance of SCM, followed by a space, followed by bar.

\section{Named Entity Paraphrasing}

In our prototype named entities include mobile phones, chargers, headsets, batteries, operating systems, applications, tariffs, manufacturers and providers. All these types of named entities have their 


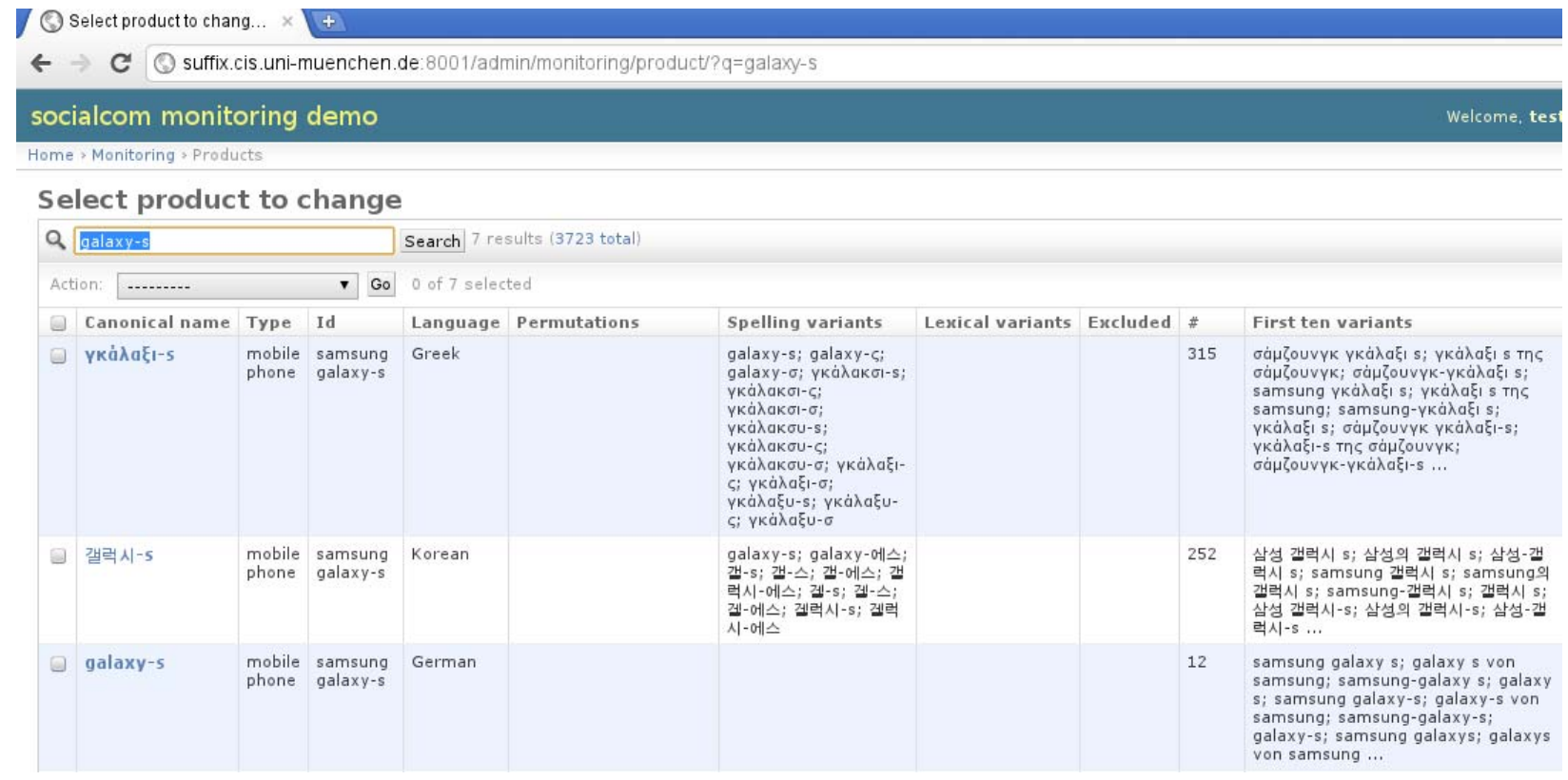

Figure 2. Samsung Galaxy S in Greek, Korean and German

own paraphrasing mechanisms. As most of our named entities are product names and as product names usually have much more variants than e.g. manufacturer names and, therefore, more interesting paraphrasing mechanisms in SCM, we will concentrate on product names in this section. New instances of SCM tailored to any other set of types of named entities can easily be created. I.e. what we are going to say about product names in the remainder of this paper also holds for organization names, EU Guidelines, and many other types of named entities, mutatis mutandis.

Our first approach to product name paraphrasing was to use paraphrasing classes. Much as verbs are inflected according to their inflection class, product names were transformed according to their paraphrasing class. Yet, paraphrasing classes had to be assigned manually and quite many classes were needed. Therefore, we decided to use a simplified system: Each product or manufacturer name is stored in a canonical form: Thus, a name of the type glofiish $g 500$ is stored in the form glofiish-g-500, even if glofiish $g-500$ or glofiish g500 should be more frequent. The minus characters tell SCM where a new part of the product name begins. A product or manufacturer name has permutations: In German o2 online-tarif has the permutation tarif-o2-online. Standard permutations are added automatically: A product or manufacturer name with three parts has the standard permutation 123. German tariff names of the type o2 online-tarif have the standard permutations 312 and 23 von 1 as in online-tarif von 02 (onlinetariff by o2), in addition. Grammar experts can add more permutations manually. Apart from their canonical name and its variants, product names can also have spelling variants. Thus, android has the spelling variants androit, antroid, antroit, andorid, adroid, andoid and andoit. (These are some of the most frequent ways android is actually spelt in the customer messages.) For each spelling variant, SCM automatically generates all paraphrases that exist according to the standard and the manually added permutations of the canonical name. I.e. the paraphrases of the mobile phone name $e$-ten glofiish- $g$ 500 include e-ten klofisch-g-500, e-ten klofisch-g 500, e-ten klofisch $g$-500, ...

Apart from spelling variants, product names can also have lexical variants. The mobile phone tct mobile $o t-v-770-a$ has the lexical variant playboy-phone. The regular permutation transformations are not applied to lexical variants. But lexical variants and their manufacturer-based variants (e.g. tct playboy-phone and playboy-phone) are, of course, paraphrases, too.

Depending on which characters are defined to be possible replacements of the minus character (the empty character, space, comma, ... ), SCM will generate a greater or smaller number of strings that actually never occur in the customer messages. But, for several reasons, overgeneration is not a problem. First, grammar experts can manually exclude variants. Secondly, most of the variants that never occur in the messages (such as tct.mobile-ot,v770.a) will hardly be used with the intent to refer to something else than the product of whose name they are claimed to be a paraphrase. 
SCM's product name paraphrasing engine is aware of the fact that many products are sold in different countries under the same or under different names. SCM stores a unique international ID for each product. Product names and their paraphrases are language specific. SCM normalizes found product names to the international ID. This way, SCM provides the data for answering questions like: Which statements are made about this mobile phone in which languages/in which social networks/in which countries/. . . ? Figure 2 shows some of the Greek, Korean and German names of the product with the international ID samsung galaxy-s.

\section{Development and Application of Grammars}

Grammar experts can create any number of content and sentiment classifiers. As already pointed out, a classifier's grammar consists of a set of positive constraints and a set of negative constraints. To classify a message, SCM simply applies the grammars of all its (content or sentiment) classifier objects to the message. Put simply, tags are assigned in the following way: If a content classifier's grammar matches, then its tag is added to the message's tag list. Sentiment classification works analogously with the exception that each message gets exactly one sentiment tag. For a classifier to match means that its positive regular expressions match and its negative regular expressions do not match or that there are no negative regular expressions. For a set of regular expressions to match means that at least one of the regular expressions matches.

Actually, the application of content classifiers is a little bit more complicated than described above. The exact procedure will be described in the next section.

Content and sentiment classifiers are language- and url-specific: A classifier has exactly one language and a set of urls. It will only be applied to messages that have the same language and that stem from one of the urls in the classifier's set of urls.

SCM supports grammar creation in various ways: All objects in SCM, such as content classifiers, sentiment classifiers, countries, languages and messages are searchable and can be filtered by various criteria. Grammar experts usually deal with messages: They apply the content classifiers they (or other grammar experts) have created (or, for short, they apply the grammar) to all or certain messages. They can then filter the result: They might want to see only the messages that have received at least one content tag. Or they might want to see the messages that have not received a sentiment tag. Or they might want to see only Korean messages, that have no content tag and are from a certain url, and so on. Grammars and constraints that contact center agents unknowingly generate can be supervised and manually corrected by grammar experts.

We think that the fact that contact center agents can invent new tags and assign new or old tags to (badly) classified messages, if they mark the strings that are supposed to justify the assignment of the tag, is a good reason for not using a statistical approach. If we used a statistical approach, human work would be necessary at some point of the development process: Some algorithm would have to be trained. In our approach, the human work is done in the customer management process. This way, two things are achieved in one step: The customer's request is answered and the classification algorithm is enhanced. An SCM instance is being enhanced while it is used. There is no need to interrupt the customer interaction in order to train SCM on new data that data specialists have created. Besides, manual intervention is much more straightforward and transparent, if a grammar of the type described above is used than it would be with a statistical algorithm. Our system is flexible in the sense that it can easily be modified in such a way that very specific requirements are met. If, e.g., a future user of SCM (a company that wants to interact with its customers) should want to assign every message that has the word hotline in it a certain tag - such as hotline problem -, then this requirement can be met by simply adding the line hotline to the positive constraints of the classifier called hotline problem.

SCM follows the DRY principle (Don't repeat yourself, see e.g. [10], page 35): Changes are only made in one place. An example: the Korean variants of the mobile phone name with the international ID google nexus-s include google nexus s, google nexus-s, nexus $s$, nexus-s, 구글 넥서스 에스, 구글 넥서스에스, 구글 넥서스 $s$, 구글 넥서스-S, 넥서스 에스, 넥서스에스, 넥서스 S, 넥서스-S, 구글 nexus $s$, 구글 nexus-s, google 넥서스에스, 구글의 넥서스에스.

This phenomenon is represented in SCM as follows: The Korean manufacturer name corresponding to the international ID google has the variants google and 구글. The Korean name for nexus-s has the variants nexus-s, 넥서스에스 and 넥서스-S. This is the only information SCM users have to store in order to make SCM generate these and many other variants. 


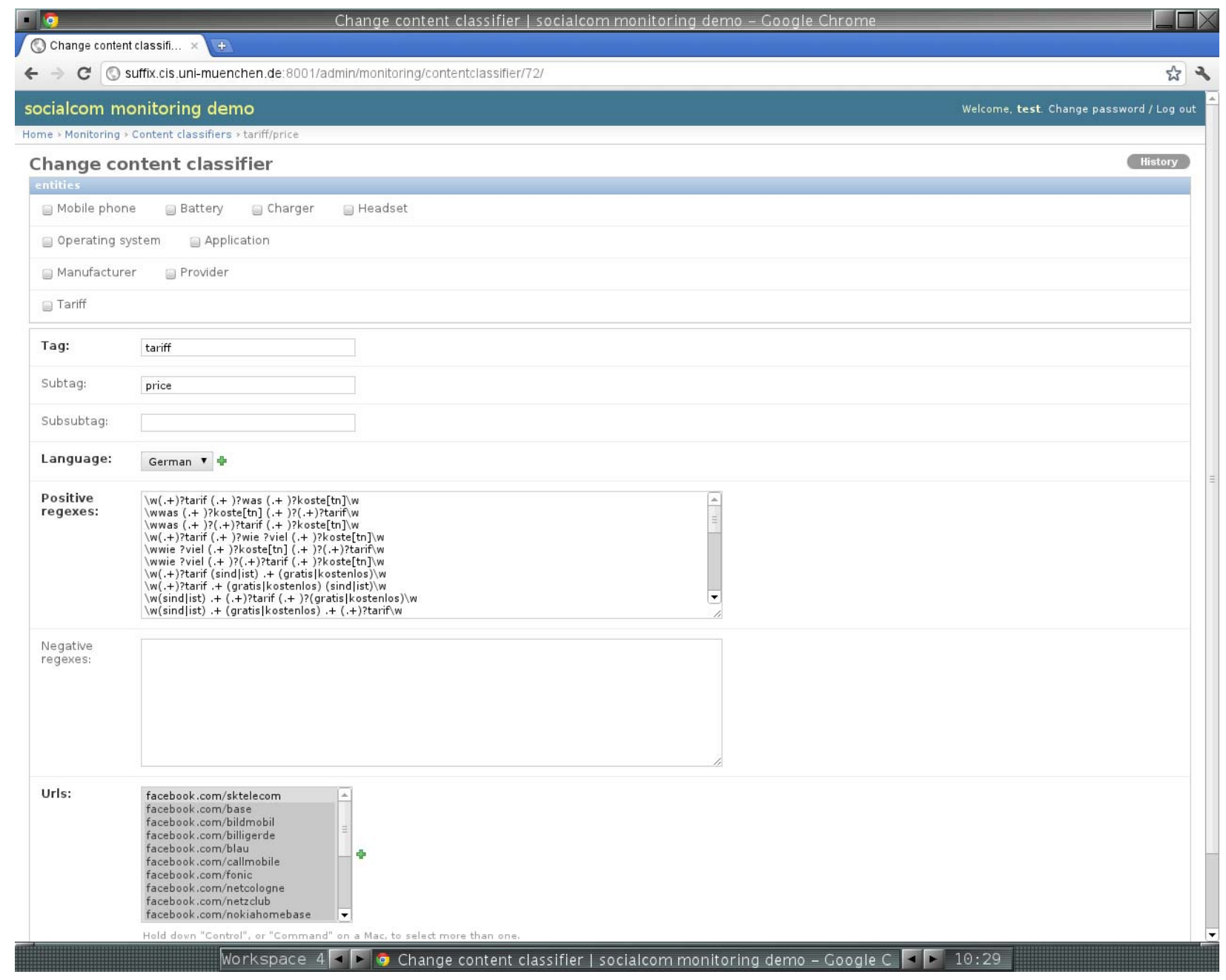

Figure 3. An unrestricted content classifier

SCM generates google nexus $s$, 구글 넥서스 $s$ and similar variants using the general rule that in any permutation of a product name any minus character may be replaced by a space character. SCM generates 넥서스에스, 넥서스 $S$ and similar variants using the general rule that the manufacturer name may be omitted. And SCM generates 구글의 넥서스에스 using the two Korean variants of the manufacturer name and the general rule that phone names can have the form $<$ manufacturer name $>$ 의 <product name $>$. (의 is a genitive affix, i.e. 구글의 넥서스에스 literally means Google's Nexus $S$ or Nexus S by Google.)

We might, of course, add the general rule to SCM that any part of a Korean product name may be spelt either with Latin or with Hangul characters - according to several sets of transliteration conventions that are used in parallel.

Any change in a manufacturer, tariff or product name object, such as the Korean mobile phone name with the international ID google nexus-s, has implications for the grammars of the message classifiers: Newly generated variants of the product name must be matched by all instances of $\backslash$ mobile_phone in all grammars. For efficiency reasons, we compile all product names, tariff names, manufacturer names, content classification grammars, sentiment classification grammars, and so on, into one single function. The compiling and reloading of this function is done in the background, so the users of SCM do not need to know anything about it. They don't even have to understand the word compile. They just need to know that SCM sometimes needs a few seconds to be able to use changed objects. We tried to design SCM such that it is easy for grammar experts to create and maintain grammars and similar entities (such as product names and manufacturer names). We prefer longterm maintainability and usability over an algorithm that might perform better in a first demo version, but that is too rigid or complicated to be adjusted to new phenomena or to be otherwise maintained. 


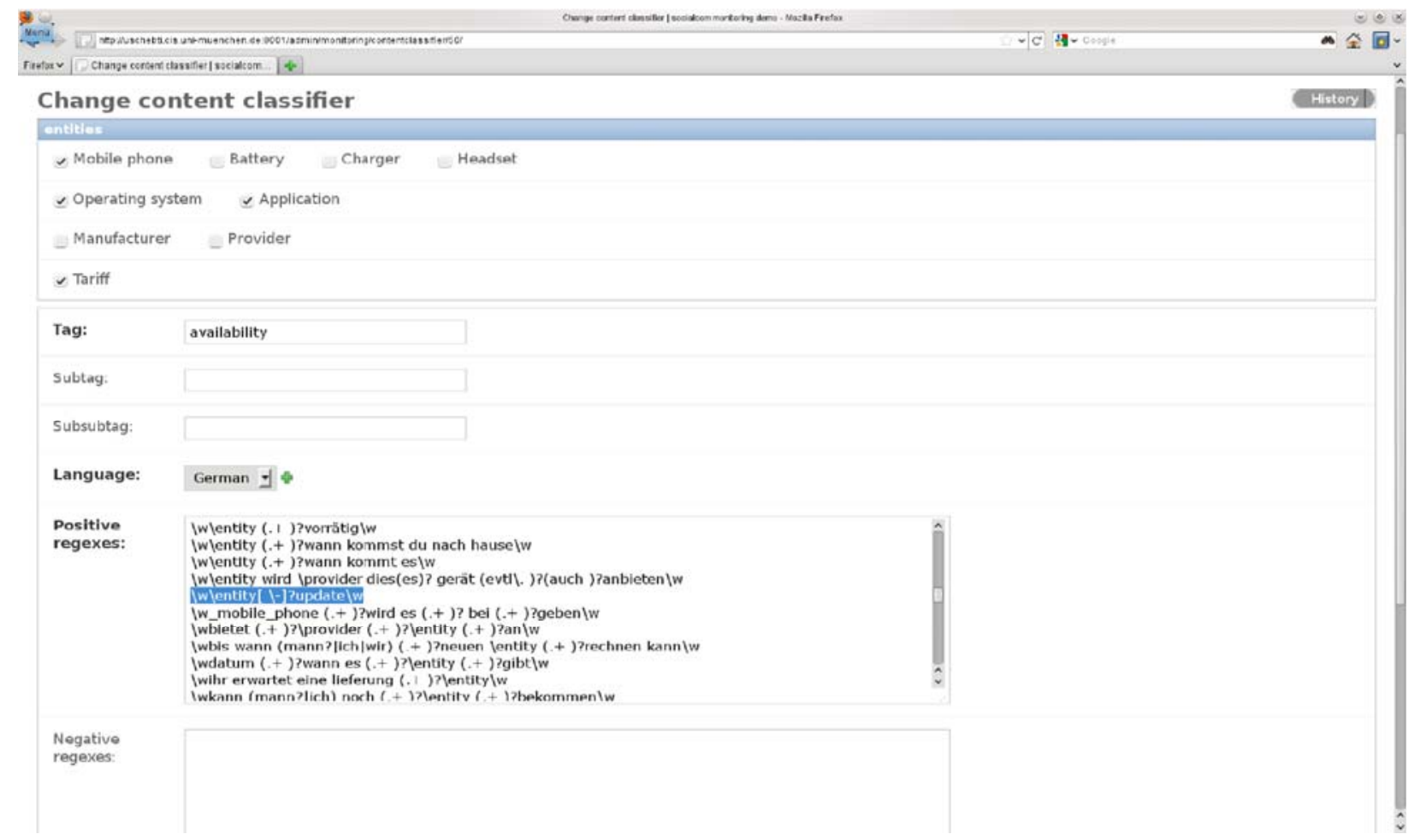

Figure 4. A restricted content classifier

\section{Content Classification}

Unlike sentiment classifiers, content classifiers come in two types: They may or may not be restricted to a set of entity types. If a content classifier is restricted to certain entity types, then a message must at least contain one instance of some of the chosen entity types in order for the content classifier to match. In the grammars of restricted content classifiers the special expression \entity refers to any instance of any of the chosen entity types. An example: The content classifier in figure 4 is restricted to the entity types mobile phone, operating system, application and tariff. This means that for this content classifier to match a message must at least mention one concrete mobile phone, operating system, application or tariff. In this grammar, the expression lentity matches any instance of any of these entity types. I.e. it would e.g. match Iphone 4.

Unrestricted content classifiers as the one shown in figure 3 do not, in general, require a message to contain any named entities. I.e. the expression lentity is meaningless in an unrestricted classifier's grammar. If a grammar author tries to use it, an error is raised. Grammar authors may use, though, expressions like \mobile_phone or t tariff in the grammars of unrestricted classifiers to require an instance of a specific type of named entities to be present in the message.

The idea of restricted content classifiers is to reuse code: We need to describe only once how customers express that some entity does not work or how they ask when some entity will be available. The more restricted content classifiers grammar authors use, the shorter will the grammar be. Yet, as not all phenomena can be described with restricted content classifiers, we need unrestricted classifiers, too.

Often grammar authors will have to create pairs of one restricted and one unrestricted content classifier that do almost the same. An example: To express that a mobile phone does not work one may either say my mobile phone does not work or, e.g., my Nexus $S$ does not work. To classify the first message, we need an unrestricted content classifier with a grammar rule of the type mobile phone .+ (not $\left.\mid n^{\prime} t\right)$ work. To classify the second message, we need a restricted content classifier with a grammar rule of the type lentity $++\left(\right.$ not $\left.\mid n^{\prime} t\right)$ work. In the second case, we would assign a tag of the type malfunction/mobile phone/google nexus-s. In the first case, we would assign a tag of the type malfunction/mobile phone. We will immediately discuss how these tags come about. 


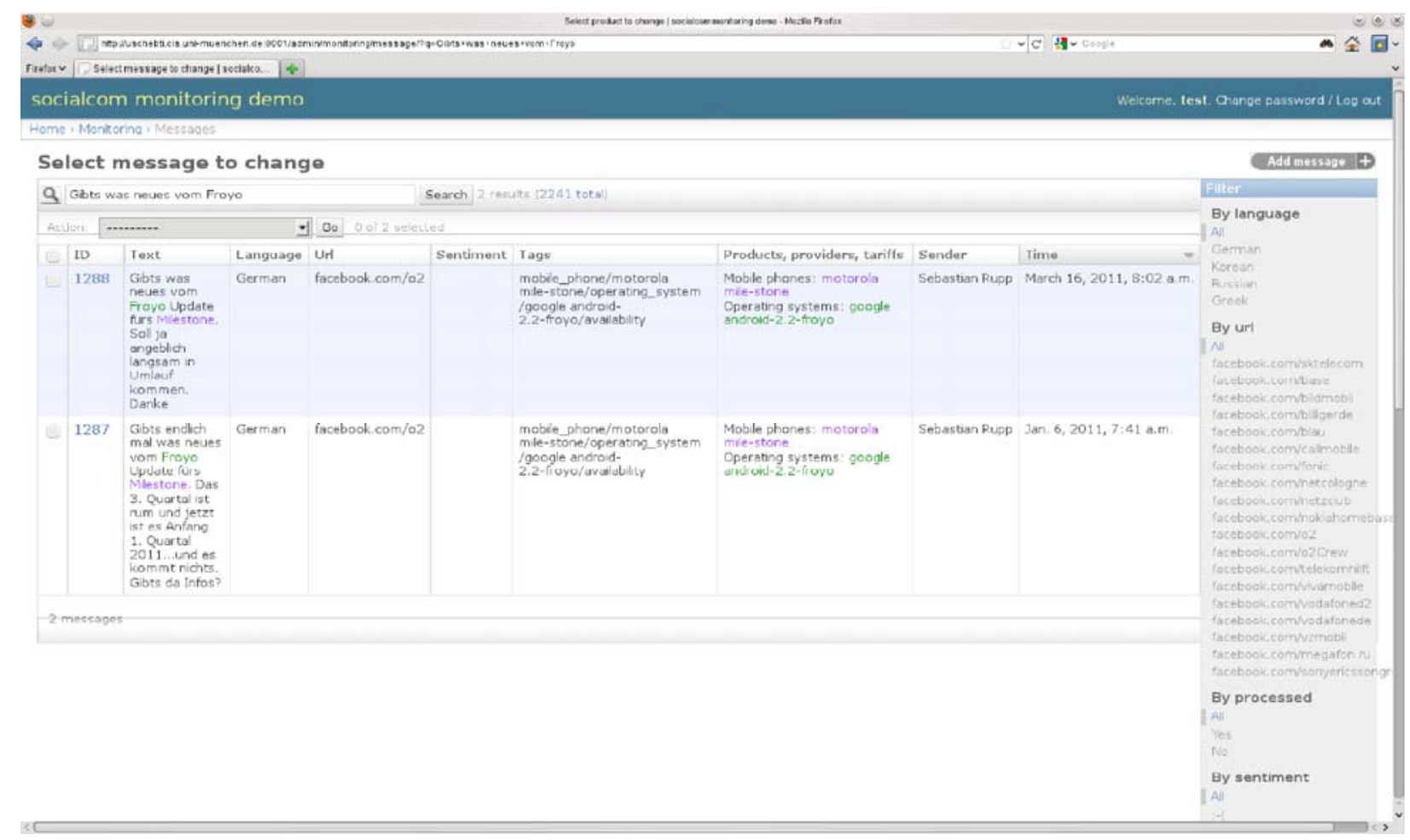

Figure 5. Messages in an SCM instance

Tags of content classifiers (restricted or unrestricted) may consist of up to three parts which we call tag, subtag and subsubtag. Only the field tag is obligatory. The three parts refer to three levels of generality. Sometimes we can only assign a rather general tag, such as network. Sometimes we might not only be able to guess that a message is about the network, but also, and more specifically, about umts. Sometimes we might even be able to guess that a message is about network, and, more specifically, about umts and, even more specifically, about the malfunction of the umts network. In this case, the content classifier with tag network, subtag umts and subsubtag malfunction would match and the tag network/umts/malfunction would be assigned. As experience taught us that more than three levels of generality (or specificity) are hardly needed, we limited the number of tag parts to three. A more specific tag part may only be present if the more general parts are present, too. Thus, an error message will be raised, if a grammar author tries to create a content classifier that has a subsubtag, but no subtag or no tag.

By using several levels of generality, we are able to distribute classified messages to contact center agents in a flexible way: A contact center agent may be an expert for a whole "branch" of the classification tree (such as network), or there may be one expert for umtsrelated issues and another expert for lte-related issues. Also, messages may be displayed and searched according to different levels of generality. And certain levels of generality may be ignored for certain tasks. In the case of unrestricted content classifiers, tag assignment is straightforward: The three tag parts are joined with slashes to form the tag to be assigned. But with resctricted content classifiers tag assignment is more complicated, as their application may involve reasoning rules.

Reasoning rules are a new and experimental feature of SCM. At the moment, grammar authors cannot create or change reasoning rules. They are not a feature of grammars or content classifiers, but of SCM. Here is an example of a very straightforward reasoning rule: Suppose a restricted content classifier with a tag such as availability or malfunction or how does it work? and with a set of entity types, such as mobile phone, headset, battery, operating system and application matches a certain message. Suppose the message contains exactly one named entity that matches the content classifier's entity types. The message might e.g. only mention the mobile phone Google Nexus $S$ and no other mobile phones and no headsets and no batteries and no .... Then it is very probable that the topic of the message was if Google Nexus $S$ is available or that a Google Nexus $S$ does not work or how the Google Nexus $S$ works, respectively. Therefore, we can assign a tag of the following type: availability/mobile phone/google nexus s (or malfunction/mobile phone/google nexus s or how does it work?/mobile phone/google nexus s). I.e. the tags that restricted content classifiers assign may contain 
entity types and concrete named entities which they get from reasoning rules.

As most messages in social networks are very short, reasoning rules work well, even if they are very simple. An example: Our prototype assigned the second message in figure 5 the tag mobile phone/motorola mile-stone/operating system/google android-2.2-froyo/availability. I.e. the system stated that the meaning of the message is whether the operating system Android 2.2 is available for the mobile phone Motorola Milestone. Instead of using slash-joined tags, we might, of course, also use formulas of predicate logic or plain English to represent "meanings" of messages. Interestingly, the sender of the message asked the same question again about two months later. But he used a slightly different wording. Our system assigned exactly the same tag to the second message. That means our system recognized the two messages as paraphrases of each other. As questions with this type of meaning ("when is X available for Y") are asked very often, they might be answered semiautomatically in the future. A contact center agent might create standard answers for certain values of $\mathrm{X}$ and $\mathrm{Y}$.

The reasoning rule necessary for correctly classifying the messages of figure 5 works roughly as follows: If the message's tag is availability and the message contains exactly one named entity of type mobile phone and exactly one named entity of type operating system, then the sender probably wants to know if (when) the operating system is (will be) available for the mobile phone. There are many similar cases: Thus, if a message's tag is availability and if it contains exactly one named entity of type application and exactly one named entity of type mobile phone, then the question is probably if this application is available for this mobile phone. Or, if a message's tag is installation and the only two named entities are of type application and operating system, then the message is probably about how to install this application on this operating system.

As these and other reasoning rules are very specific and domain-dependent, they should not be part of SCM, but of concrete SCM instances. We might implement this in the future. There are basically two ways to implement it: An easy and ugly way and a difficult and beautiful way. We chose the easy and ugly way for our current SCM prototype. We made a copy of SCM - of the "pure", the domain independent SCM - and added domain-specific reasoning rules to it. This can also be done for other domains. The difficult and beautiful way would be to enable grammar authors - or other agents - to create domain specific reasoning rules for instances of SCM via the web interface. This solution would be beautiful in so far as it does not involve copying and adapting of source code. There would be one and only one SCM and many different instances of it. Reasoning rules would be data just like product names or content classifiers. Yet, experience teaches us that it is often not efficient to create systems that are overly beautiful. In our case, a disadvantage would be that grammar authors would have to learn one more feature of the system - and a very complicated feature. Besides, it is not clear how the abstract form of reasoning rules should look like. Which elements does it take to create a reasoning rule? Which relations between these elements can hold? How are grammar authors supposed to describe the reasoning rules?

One possibility to give grammar authors responsibility for reasoning rules would be the following: Grammar authors might use tags as predicates and entity types as arguments. Thus, they might write a grammar for the topic availability and choose an arity for this topic, e.g. two. Then they might write rules of the type Is \} 1 \text { available for } $\backslash 2$ ? and decide that - in this specific content classifier $-\backslash 1$ may refer to an application and $\backslash 2$ to a mobile phone or an operating system, or, respectively, $\backslash 1$ might refer to a battery or a headset and $\backslash 2$ might refer to a mobile phone (but, obviously, not to an operating system). This way we could still share code, but hand over responsibility for reasoning to the grammar authors. And we would have more precise and powerful grammar rules. Yet, it would also make the system much more complicated - for its authors as well as for its users.

\section{Conclusion}

In this article, we presented SCM, a system for web monitoring and customer interaction. We tried to show how these tasks can be automatized to a large degree without making the system too complicated and intransparent. Our system is highly flexible and modular, because it has a software agent for each of the entities involved in the monitoring and customer interaction process. In SCM companies are mapped to company objects, products are mapped to product objects, text types are mapped to text type objects, and so on. Each of the objects can easily be modified by grammar experts. SCM makes sure that any change in an object is communicated to all the other objects that must know about it.

We hope to be able to enhance SCM in several ways in the future. The most important enhancements are perhaps the following two. On one hand, we should find a general and domain-independent solution for creating reasoning rules. On the other hand, SCM should be able to learn more things automatically. As many of SCM's grammars use special terms for matching certain types of expressions, such as $\backslash$ mobile phone, \charger, \headset, $\backslash$ tariff, \software, \operating_system or $\backslash$ battery, minor code changes will suffice to enable SCM to find unknown mobile phone, charger or headset names in customer messages: As our grammars are designed for classifying messages of 
specific types, they contain very good contexts of named entities. Thus, a grammar that describes messages written by customers who want to install a certain software on a certain mobile phone will probably contain good contexts for software and mobile phone names.

\section{Acknowledgment}

The Socialcom project was funded by the German Federal Ministry of Economics and Technology.

\section{References}

[1] Gryc, W., and Moilanen, K. (2010) 'Leveraging Textual Sentiment Analysis with Social Network Modelling: Sentiment Analysis of Political Blogs in the 2008 U.S. Presidential Election', in Proceedings of the From Text to Political Positions Workshop (T2PP 2010), Vrije Universiteit, Amsterdam, April 9-10 2010; http://users.ox.ac.uk/ wolf2244/gryc_moilanen_t2pp_2010_f inal.pdf (26 July 2011).

[2] Krauss, J., Nann, S., Simon, D., Fischbach, K., and Gloor, P. A. (2008) 'Predicting Movie Success and Academy Awards Through Sentiment and Social Network Analysis', ECIS European Conference on Information Systems.

[3] Pal, J. K., and Saha, A. (2010) 'Identifying Themes in Social Media and Detecting Sentiments', HP Laboratories, Tech. Rep. HPL-2010-50; http://www.hpl.hp.com/techreports/2010/HPL-2010-50.pdf (26 July 2011).

[4] Mishne, G. (2007) Applied Text Analytics for Blogs, University of Amsterdam, Enschede/Netherlands.

[5] Jijkoun, V., Khalid, M. A., Marx, M., and de Rijke, M. (2008) 'Named entity normalization in user generated content', in Proceedings of the second workshop on Analytics for noisy unstructured text data, ser. AND '08: New York, NY, USA, ACM, pp. 23-30.

[6] Raghavan, H., and Allan, J. (2005) 'Proper Names and their Spelling Variations in Automatic Speech recognition output', University of Massachusetts, IR 361.

[7] Gross, M. (1989) 'The Use of Finite Automata in the Lexical Representation of Natural Language', in Proceedings of the LITP Spring School on Theoretical Computer Science, Electronic Dictionaries and Automata in Computational Linguistics: Springer-Verlag, London, pp. 34-50.

[8] Browne, R., Clements, E., Harris, R., and Baxter, S. (2009) 'Business and consumer communication via online social networks: a preliminary investigation', in Australian and New Zealand Marketing Academy (ANZMAC) Conference; http://www.duplication.net.au/ANZMAC09/papers/ANZMA C2009-328.pdf.

[9] Friedrich, G., and Shchekotykhin, K. (2006) 'NameIt: Extraction of product names', Data Mining Workshops, International Conference on: vol. 0, pp. 29-33.

[10] Colbaugh, R., and Glass, K. (2010) 'Estimating sentiment orientation in social media for intelligence monitoring and analysis', in ISI: Vancouver, BC, Canada, pp. 135-137.

[11] Locke, B., and Martin, J. (2009) 'Named Entity Recognition: Adapting to Microblogging', University of Colorado.

[12] Bothos, E., Apostolou, D., and Mentzas, G. (2010) 'Using Social Media to Predict Future Events with Agentbased Markets’, IEEE Intelligent Systems, vol. 99.

[13] Laboreiro, G., Sarmento, L., Teixeira, J., and Oliveira, E. (2010) 'Tokenizing Micro-Blogging Messages using a Text Classification Approach', in AND’10: Toronto, Ontario, Canada.

[14] Kaufmann, M. (2010) 'Syntactic Normalization of Twitter Messages', studies, pp. 1-7; http://www.cs.uccs.edu/_kalita/work/reu/REUFinalPapers20 10/Kaufmann.pdf (26 July 2011).

[15] Key-Sun, J.-S. N., and sun Choi, K. (1997) 'A Local Grammar-based Approach to Recognizing of Proper Names in Korean Texts', in Proceedings of the Fifth Workshop on Very Large Corpora: ACL/Tsing-hua University/Hong-Kong University of Science and Technology, pp. 273-288.

[16] Kozareva, Z. (2006) 'Bootstrapping named entity recognition with automatically generated gazetteer lists', in Proceedings of the Eleventh Conference of the European Chapter of the Association for Computational Linguistics: Student Research Workshop, ser. EACL '06: Stroudsburg, PA, USA, Association for Computational Linguistics, pp. $15-21$.

[17] Volk, M., and Clematide, S. (2001) 'Learn - Filter Apply - Forget. Mixed Approaches to Named Entity Recognition' in Proceedings of the 6th International Workshop on Applications of Natural Language to Information Systems: GI, pp. 153-163.

[18] Kaplan, A. M., and Haenlein, M. (2010) 'Users of the world, unite! The challenges and opportunities of Social Media': Business Horizons, vol. 53, pp. 59-68. 